\title{
Point diffraction interferometry for the rotational symmetry analysis in fish lenses
}

\section{Interferometría de difracción por punto para el análisis de la simetría de rotación en cristalinos de peces}

\author{
Antía Blanco Bernárdez ${ }^{(1,2, S, E)}$, Ana Gargallo Fernández(1,S), Eva Acosta Plaza(1) \\ 1. Departamento de Física Aplicada (Área de Óptica), Universidade de Santiago de Compostela, Facultade \\ de Óptica e Optometría (Campus Vida) 15782 Santiago de Compostela, Spain. \\ 2. Óptica Bueu. Pazos Fontenla, 5136930 Bueu Pontevedra, Spain. \\ (*) Email: antia.blanco.bernardez@gmail.com S: miembro de SEDOPTICA / SEDOPTICA member \\ Received / Recibido: 19/09/2014. Revised / Revisado: 25/11/2014. Accepted / Aceptado: 26/11/2014. \\ DOI: http://dx.doi.org/10.7149/OPA.47.4.321
}

\begin{abstract}
:
It is generally assumed that teleost fish lenses are spherically symmetrical. Our purpose is to evaluate this symmetry by means of point diffraction interferometer (PDI) which is an accurately technique to evaluate very aberrated optical elements as eye lenses. Several lenses of two different fish were evaluated in vitro with a PDI, they were placed over a ring inside a glass cell filled with Medium 199. Measurements were performed for ten different positions of the lens, from a position with equatorial plane orthogonally to optical axis of the system to a position with equatorial plane quasi-parallel with optical axis. Based on the assumption of a spherically symmetrical refractive index, they should yield radially-symmetric interferograms regardless of their position. With PDI measurements we are able to test visually and numerically this assumption. We found that only when the fish lens is positioned with equatorial plane orthogonally to the optical axis of the system shows a quasi-spherical symmetry. This technique allows quantify the deviation from the spherical symmetry commonly assumed for teleost fish lenses.
\end{abstract}

Key words: Point Diffraction Interferometry, Interferometer, Fish Lenses.

\section{RESUMEN:}

Generalmente se asume que los cristalinos de peces son esféricos. Nuestro objetivo es evaluar esa simetría utilizando un interferómetro de difracción por punto (IDP), que ha demostrado ser una técnica muy precisa para la caracterización de elementos ópticos muy aberrados como los cristalinos. Se estudiaron varios cristalinos in vitro de dos especies diferentes de peces. Una vez extraídos del globo ocular se colocaron dentro de una cubeta con Medium 199 sobre una arandela especialmente diseñada para el estudio, que permitía la toma de los interferogramas en diez posiciones diferentes, desde una posición con el plano ecuatorial ortogonal al eje óptico hasta una posición casi paralela. Basándonos en una geometría esférica y una distribución homogénea del gradiente de índice de refracción los interferogramas obtenidos deberían ser radialmente simétricos independientemente de su posición. El IDP nos permite verificar visual y numéricamente esta simetría. Hemos encontrado que sólo cuando el cristalino de pez tiene el plano ecuatorial ortogonal al eje óptico, el sistema muestra una simetría casi esférica. Esta técnica permite cuantificar la desviación de esa esfericidad que comúnmente se asume para estos peces.

Palabras clave: interferometría de Difracción por Punto, Interferómetro, Cristalinos de Peces.

\footnotetext{
${ }^{\mathrm{E}}$ During the development of the work, coauthor A. Blanco Bernárdez was student of the Optics and Optometry Grade of the University of Santiago de Compostela (Spain).
} 


\section{REFERENCES AND LINKS / REFERENCIAS Y ENLACES}

[1] R. H. Kroger, K. A. Fritsches, E. J. Warrant, "Lens optical properties in the eyes of large marine predatory teleosts", J. Comp. Physiol. A 195, 175-182 (2009). DOI

[2]. E. Acosta, D. Vazquez, L. R. Castillo, "Analysis of the optical properties of crystalline lenses by pointdiffraction interferometry", Ophthal. Physiol. Opt. 29, 235-246 (2009). DOI

[3]. A. Gargallo, J. Arines, E. Acosta, "Lens aberrations and their relationship with lens sutures for species with Y-suture branches", J. Biomed. Opt. 18, 025003 (2013). DOI

[4]. W. S. Jagger, "The optics of the spherical fish lens", Vis. Res. 32, 1271-1284 (1992). DOI

[5]. R. H. Kroger, M. C. Campbell, R. D. Fernald, H. J. Wagner, "Multifocal lenses compensate for chromatic defocus in vertebrate eye", J. Comp. Physiol. A 184, 361-369 (1999). DOI

[6]. E. Acosta, S. Chamadoira, R. Blendowske, "Modified point diffraction interferometer for inspection and evaluation of ophthalmic components", J. Opt. Soc. Am. A 23, 632-637(2006). DOI

[7]. E. Acosta, S. Chamadoira, S. Bosch, S. Vallmitjana, "Wavefront local curvature evaluation by multiple point diffraction interferometry", Opt. Pura Apl. 46, 157-163 (2013). DOI

\section{Introduction}

Los ojos de los vertebrados son parecidos en diseño a una cámara fotográfica. Sin embargo, mientras que el objetivo de una cámara está constituido por un variable número de lentes que corrigen las diferentes aberraciones ópticas, en los ojos de vertebrados esta función la desempeñan únicamente dos lentes, córnea y cristalino.

En el caso de los animales acuáticos, el poder dióptrico de la córnea es prácticamente despreciable debido a que está en contacto por ambas caras con líquidos de índices de refracción muy similares (agua y humor acuoso). Por lo tanto, en estos peces la única lente responsable del enfoque y de la calidad óptica del sistema es el cristalino. Esto significa que estudiando las características ópticas de los cristalinos de estos animales podemos conocer con cierta precisión la calidad del sistema, si bien es cierto que la calidad de la visión final vendrá dada por la integración a nivel sensorial de la imagen proyectada en la retina.

Comúnmente se asume que los cristalinos de peces teleósteos presentan una forma esférica [1]. Este estudio trata de valorar si los cristalinos de dos especies diferentes de teleósteos presentan o no simetría de rotación. Para ello se utiliza un interferómetro de difracción por punto (IDP) modificado. Esta técnica ha sido utilizada recientemente para la caracterización óptica de cristalinos de peces [2] y mamíferos [2,3], demostrando una gran precisión en la medida de elementos ópticos altamente aberrados.

\section{Material y métodos}

\section{2.a. Cristalinos}

Se examinaron con el IDP siete cristalinos de Pagellus acarne (besugo) y dos de Scorpaena scrofa (cabracho), extraídos pocas horas después de la captura.

Los cristalinos de estos peces presentan un gradiente de índice que compensa la aberración esférica longitudinal que presentan las lentes esféricas. La distribución de este gradiente disminuye desde el interior hacia el exterior [4].

Por otro lado, cabe destacar que, como método para compensar la aberración cromática longitudinal, estas lentes presentan zonas multifocales que focalizan selectivamente las longitudes de onda sobre la retina [5].

\section{2.b. Interferómetro de difracción por punto}

El IDP se compone de una lámina semitransparente con un pequeño orificio circular en el centro de la misma. Al iluminar la lámina, parte de la onda se difractará por el orificio y, de acuerdo al principio de Huygens, la onda que sale a través de este agujero (si es suficientemente pequeño) será una onda esférica que actuará como onda de referencia; otra parte de la onda atravesará la lámina semitransparente y portará las aberraciones presentes en el sistema óptico a estudiar. 
El IDP es por lo tanto un interferómetro de camino común en el que las franjas de interferencia son el resultado de las variaciones de fase entre la onda examinada y la onda esférica de referencia (Fig. 1).

El interferómetro utilizado en el estudio difiere del IDP original en el tamaño del orificio de la lámina semitransparente $[2,3,6,7]$. En este caso, la apertura es mayor que el radio del disco de Airy. Esto significa que la onda de referencia a la salida de la lámina no será tan perfecta, lo que conlleva una pérdida de visibilidad en las franjas periféricas, así como una pérdida de sensibilidad. Sin embargo, gracias a esto conseguimos un gran rango dinámico.

El mayor tamaño del orificio conlleva transmitancias más altas, lo que facilita enormemente la alineación del dispositivo y la detección de las franjas en el plano de observación. Esto es imprescindible para la observación de tejidos orgánicos muy aberrados como es el caso de un cristalino.

\section{2.c. Diseño experimental}

El cristalino, inmerso en Medium 199 dentro de una cubeta y sobre una arandela, es iluminado con una onda plana monocromática (633 nm). La lámina del IDP se sitúa lejos del foco del cristalino, lo que permite obtener en una pequeña zona central de la lente un interferograma bien contrastado y por tanto valores precisos de la fase dentro de dicha zona. Se realizaron medidas en 10 posiciones diferentes, rotando el cristalino desde una posición con el plano ecuatorial ortogonal al eje óptico a casi paralelo (Fig. 3). Una cámara CCD capturaba los interferogramas en el plano de observación y otra cámara la posición del cristalino.

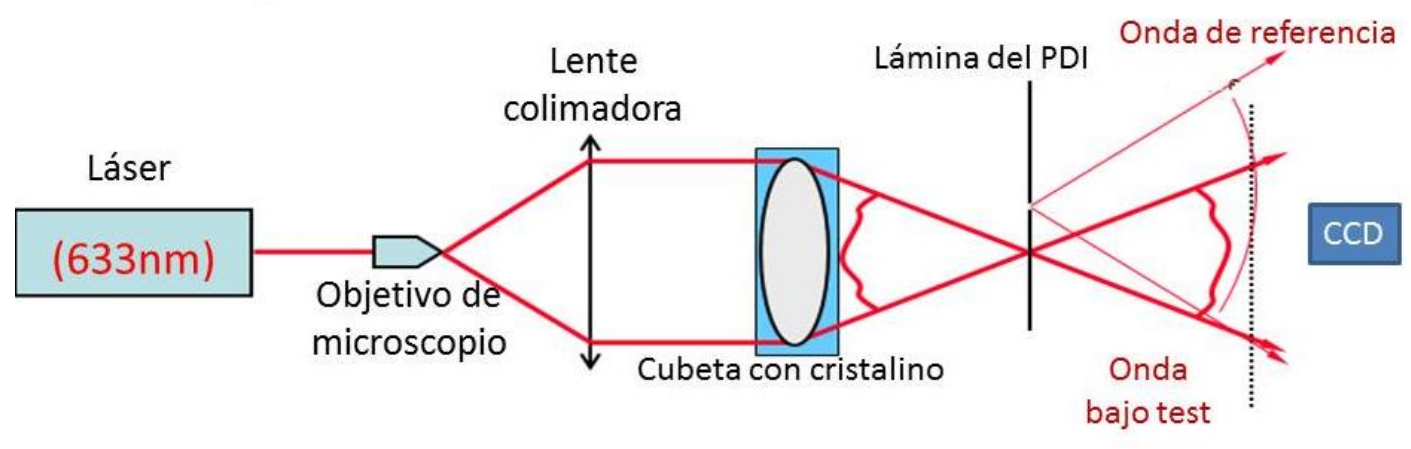

Fig. 1. Esquema del IDP.
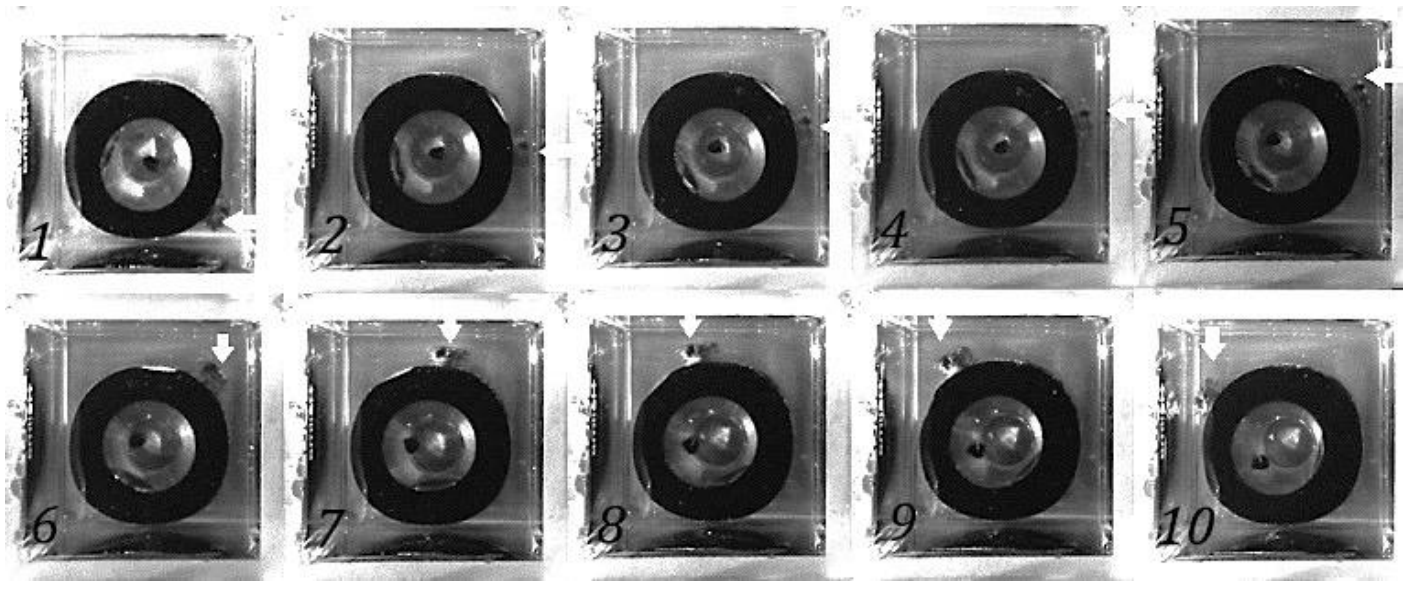

Fig. 2. De izquierda a derecha y de arriba abajo las 10 posiciones del cristalino para el besugo 4 . El giro se aprecia por el cambio de posición del tornillo (indicado con las flechas) insertado en la arandela que sostiene el cristalino. 


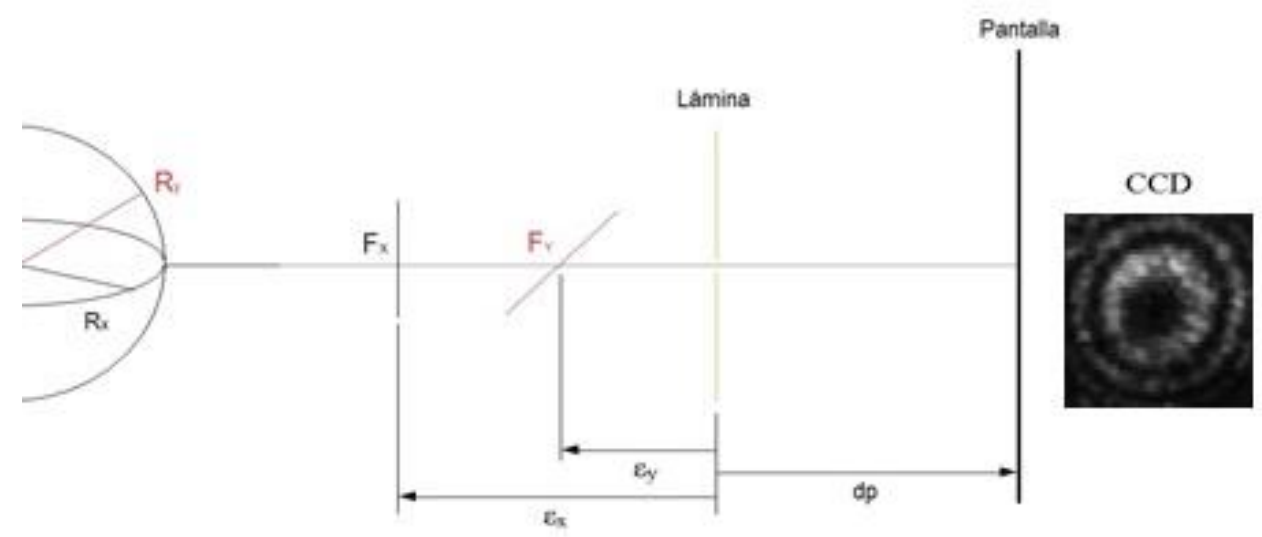

Fig. 3. Esquema de focales e interferograma de una lente esfero-cilíndrica.

\section{2.d. Teoría}

Los interferogramas se analizan en una pequeña zona del plano de medida. Si se asume que en esta región la fase se puede aproximar por una onda esferoclíndrica, ésta vendrá pues caracterizada por dos radios de curvatura. Denominaremos $R_{x}$ al radio de curvatura horizontal (en la dirección del ancho de la cubeta) y $R_{y}$ al vertical (altura de la cubeta). Debido a la forma del frente de onda a la salida de esta lente, tendremos dos líneas focales, una línea focal vertical denominada $F_{x}$, que estará a una distancia $\varepsilon_{x}$ de la lámina del IDP, y una línea focal horizontal denominada $F_{y}$, que estará a una distancia $\varepsilon_{y}$ de la lámina perforada (Fig. 3).

Las elipses que forman el interferograma se pueden considerar como la interferencia de dos ondas, la esférica de referencia, con origen en el agujero de la lámina, y la que procede del cristalino, que asumiremos puramente astigmática dentro de la pequeña región de análisis. La fase del término interferencial viene dada por:

$$
\begin{gathered}
\phi(x, y)=\frac{\pi}{\lambda}\left[\left(\frac{x^{2}}{\varepsilon_{y}+d p}+\frac{y^{2}}{\varepsilon_{y}+d p}\right)-\right. \\
\left.-\frac{x^{2}+y^{2}}{d p}\right]
\end{gathered}
$$

donde $d p$ denota la distancia de la lámina al plano de observación.

En la Fig. 2 se muestra un interferograma obtenido para una posición arbitraria del cristalino donde se observan las franjas de interferencia elípticas. Si una de las líneas focales se aproxima al plano de la lámina (por ejemplo cuando $\varepsilon_{y}$ tiende a cero), el eje de la elipse (en este caso el eje $x$ ) aumenta.

Si $x$ e $y$ denotan los radios de la elipse que forma el primer anillo oscuro del patrón de interferencia, horizontal y vertical respectivamente tendremos [6]:

$$
\begin{gathered}
\frac{\pi}{2}=\frac{\pi}{\lambda}\left[\left(\frac{x^{2}}{\varepsilon_{y}+d p}+\frac{y^{2}}{\varepsilon_{y}+d p}\right)-\right. \\
\left.-\frac{x^{2}+y^{2}}{d p}\right]
\end{gathered}
$$

Para analizar automáticamente todos los datos a partir de las imágenes obtenidas con la cámara CCD del plano de interferencia, se utilizan programas específicos desarrollados con Mathcad para procesar y analizar los interferogramas. Para el cálculo de los ejes de las elipses, y de aquí obtener $\varepsilon_{x}$ y $\varepsilon_{y}$, se tomó siempre el primer anillo oscuro del interferograma. Es de destacar además, que en un primer momento se puntea manualmente este anillo y dichos puntos son procesados posteriormente por el programa para buscar la elipse que mejor se ajuste a ellos mediante un ajuste de mínimos cuadrados.

\section{Resultados y discusión}

Como hemos indicado anteriormente, se tomaron 10 interferogramas de cada cristalino, uno por cada posición del tornillo que se observa en la Fig. 1. A medida que se gira el cristalino, las franjas se hacen menos circulares y se vuelven 
más elípticas, por lo que la elipticidad (que podemos aproximar como $\varepsilon_{y} / \varepsilon_{x}$, dado que $d p>>\varepsilon_{x}$ y $d p>>\varepsilon_{y}$ ) varía según el plano ecuatorial se encuentre o no perpendicular al eje óptico (Fig. 4).

La muestra utilizada era heterogénea tanto en número de ejemplares por especie como en tamaño. Por ello, dividimos la muestra en 3 grupos. El primer grupo está constituido por tres besugos de mayor tamaño y por tanto de mayor edad; el segundo grupo lo forman los 4 besugos restantes, con cristalinos más pequeños y el tercer grupo está formado por los 3 cabrachos con cristalinos de tamaños similares a los del grupo 2. A partir de aquí, se analizaron los resultados comparando los tres grupos.

En la Fig. 5 se observa cómo los valores de la elipticidad del primer anillo (mínimo),tanto la máxima como mínima obtenida para los tres primeros besugos (grupo 1)es superior a la de los cuatro siguientes, correspondientes a besugos más pequeños (grupo 2). Los cabrachos (grupo 3), con cristalinos de tamaños similares a los tres primeros besugos, presentan elipticidades menores que éstos pero mayores que las de los besugos más pequeños.

Los resultados parecen indicar que los cristalinos de menor tamaño son más simétricos

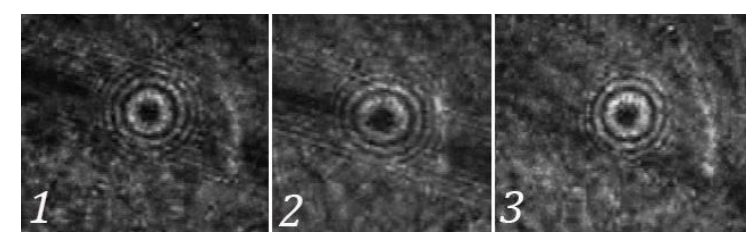

Fig. 4. Tres interferogramas de un mismo cristalino tomado en diferentes posiciones, se puede observar como las franjas cambian el tamaño de las franjas y su elipticidad.

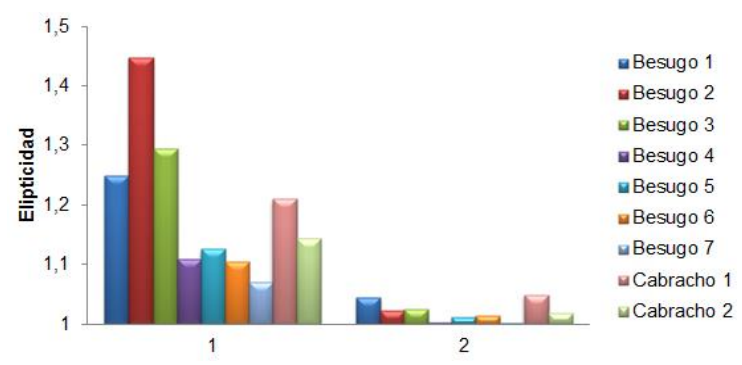

Fig. 5. Representación de los valores máximo $(\mathrm{M}, 1)$ y mínimo (m, 2) de elipticidad para cada cristalino. que los cristalinos más grandes, lo cual podría ser debido a que en los cristalinos grandes la mayor cantidad de fibras acumuladas hacia la superficie acentúe pequeñas deformidades o bien que la forma de disposición de las fibras en relación con la sutura provoque una disposición más laxa en una dirección que en otra. Parasaber si esta diferencia entre máximos y mínimos de elipticidad se corresponde también con una diferencia en la elipticidad a lo largo de superficie estudiada, en la Fig. 6 se representaron todos los valores de la elipticidad obtenidos en cada posición y cada cristalino, y por grupos. Lo que se denomina orden de la medida equivale aproximadamente a 15-20 grados.

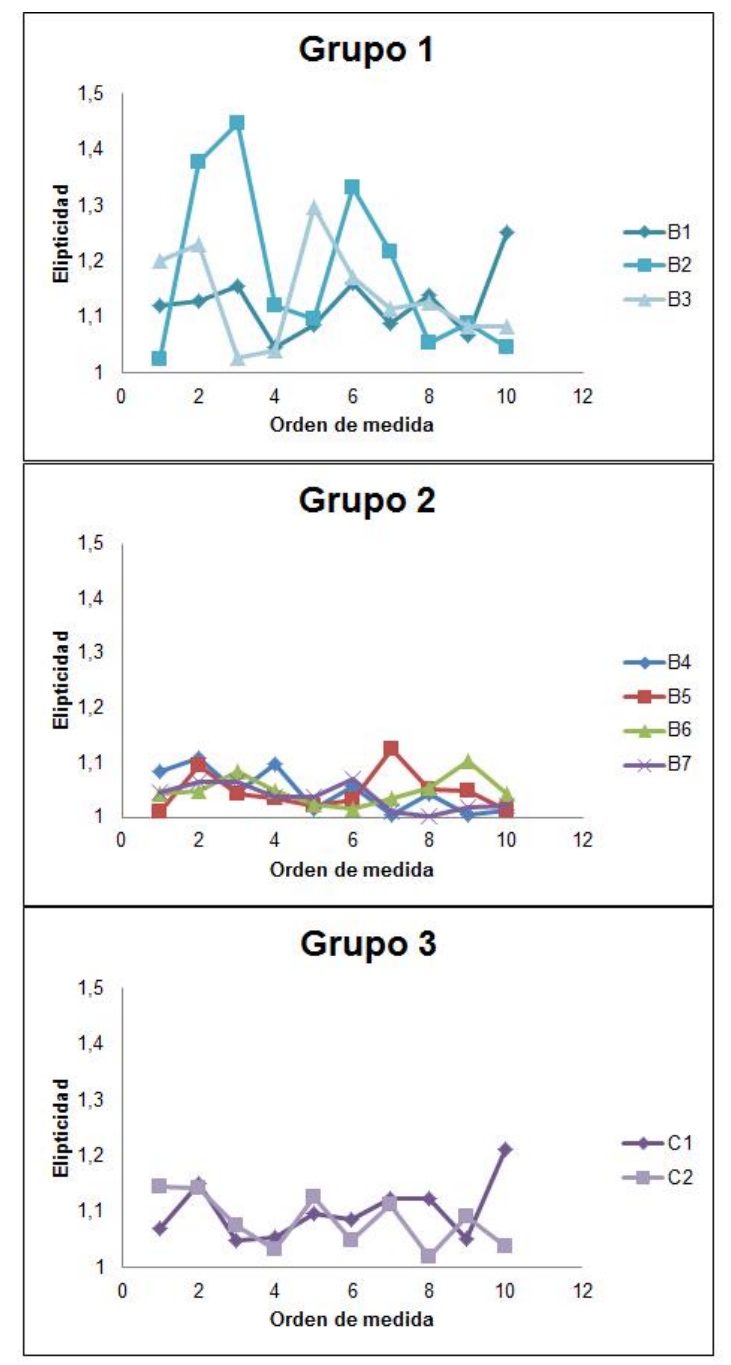

Fig. 6. Representación de la elipticidad para cada cristalino en función de la medida. 
Es importante destacar que los datos experimentales de la Fig. 6 están representados en función del orden de realización de la medida y los cristalinos fueron colocados en la cubeta sin ninguna referencia, por lo que la posición de la sutura en cada medida es aleatoria. Teniendo esto en cuenta, la disposición de los datos en tres grupos muestra una clara diferencia. Se observa que los cristalinos incluidos en un grupo se comportan de forma similar al resto de sus semejantes, mostrando una mayor variabilidad y magnitud en la elipticidad los besugos más grandes (grupo 1) seguidos por los cabrachos (grupo 3) y siendo el grupo de besugos más pequeños (grupo 2) el que muestra valores más similares entre ellos así como menor magnitud de la elipticidad a lo largo de todas las medidas.

En la Fig. 7 se muestra la relación entre la elipticidad y la diferencia que existe entre $\varepsilon_{x} \mathrm{y} \varepsilon_{y}$, es decir, separación entre las focales.

Conforme aumenta el nivel de elipticidad, es de esperar que las focales estén más separadas entre sí, por lo que esta diferencia debe aumentar de forma directamente proporcional a la magnitud de la elipticidad. En la figura 6 se comprueba que esto es así para las elipticidades pequeñas e intermedias, $\sin$ embargo, y sobre todo en los grupos 1 y 3, existen datos que no cumplen esta norma, como es el caso de los últimos valores de los besugos 2 y 3 y de los cabrachos. Estas anomalías podrían ser debidas a interferogramas "sucios" que aumentan el error de medida.

Otra evidencia importante a destacar de la Fig. 7 es que la diferencia $\varepsilon_{x}-\varepsilon_{y}$ siempre es positiva. Teniendo en cuenta que $\varepsilon_{x}$ es la distancia de la lámina a la focal proporcionada por el radio de curvatura horizontal y $\varepsilon_{y}$ la distancia de la lámina a la focal de la curvatura en $\mathrm{y}$, se deduce que la focal perteneciente a la curvatura horizontal está más cerca del cristalino y por lo tanto es más curva que la vertical. Esto quiere decir que todos los cristalinos estudiados estaban ligeramente más aplanados en el eje vertical que en el horizontal. En la Fig. 8 están representados los valores $\varepsilon_{x} \mathrm{y}$ $\varepsilon_{y}$ para cada medida y cada cristalino. Lo que se observa, sobre todo cuando nos acercamos a los valores medios de elipticidad y altos es que es el valor $\varepsilon_{y}$ el que muestra una mayor tendencia hacia valores menores. Esto coincide con lo que habíamos deducido en relación a la figura anterior, si $\varepsilon_{y}$, que es la distancia de la focal de la curvatura vertical, es menor, quiere decir que la distancia al cristalino es mayor, es decir, que la curvatura vertical se hace más plana conforme aumenta la elipticidad.

Se observan además diferencias entre las gráficas de los cristalinos pertenecientes a los distintos grupos. En este caso, se aprecian unos valores ligeramente menos variables en los cabrachos y en los besugos del grupo 1 que en los besugos del grupo 2. Esto podría deberse a que el grupo 2 presentaba elipticidades mucho menores, lo cual provoca que sus interferogramas centrales sean en muchos casos

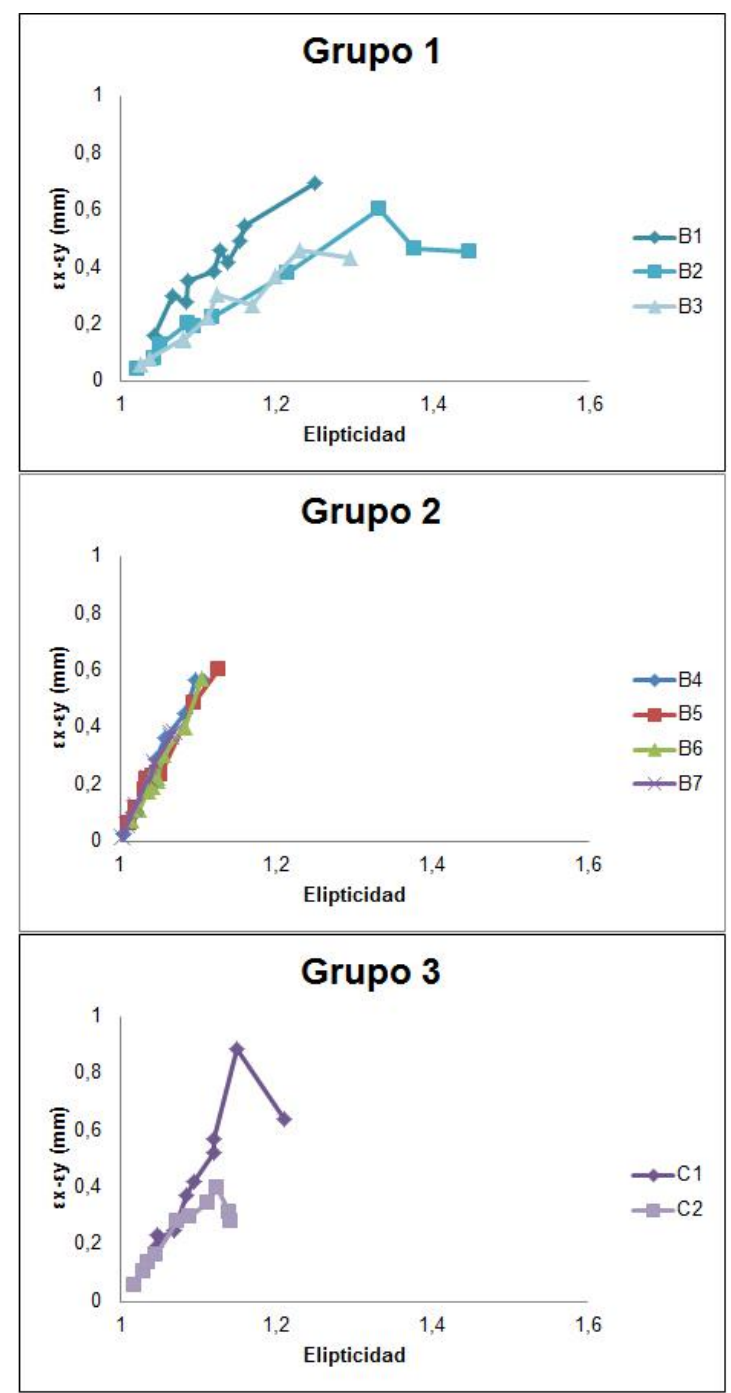

Fig. 7. Representación de la diferencia $\varepsilon_{x}-\varepsilon_{y}$ entre frente a la elipticidad. 


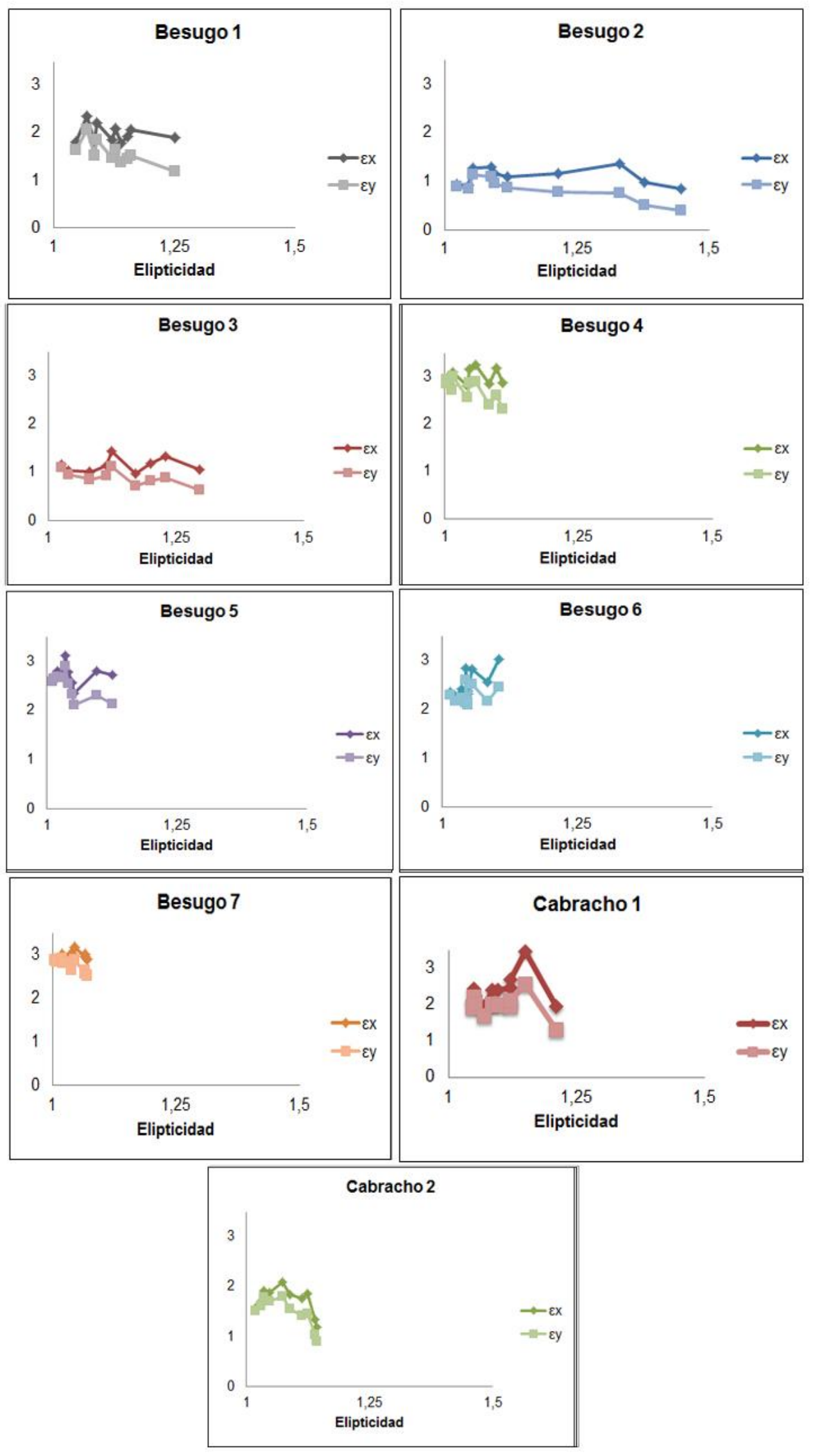

Fig. 8. Representación gráfica de $\varepsilon_{x}$ y $\varepsilon_{y}$ (en mm) para cada cristalino. 
muy cercanos a una circunferencia. Por esto, pueden ser más vulnerables a un error de medida ya que uno de los pasos en el procesado de las imágenes consiste en puntear manualmente la primera franja oscura del interferograma para que luego un programa informático analice la figura geométrica que se forma a partir de estos puntos.

\section{Conclusiones}

Basándonos en una forma esférica y una distribución simétrica del gradiente de índice de refracción, los cristalinos de peces deberían producir interferogramas radialmente simétricos indiferentemente de su posición. Esta técnica permite cuantificar la desviación de esa simetría esférica comúnmente asumida para los peces teleósteos. De esta manera, se observa que los cristalinos del Pagellus acarne y del Scorpaena scrofa no son rotacionalmente simétricos. Pequeños giros suponen un error de medida que podría ser importante, por lo que su correcto posicionamiento es esencial. Ambas especies presentan aplanamiento de la curvatura vertical con la rotación, lo que puede deberse a que la disposición de las fibras en relación con la sutura provoque una disposición más laxa en una dirección que en otra, lo que podría suponer una influencia de este tipo de sutura umbilical en las aberraciones del cristalino, como ocurre en otras configuraciones histológicas de las fibras cristalinianas, como las suturas en Y.

\section{Agradecimientos}

Este trabajo ha sido posible gracias al Ministerio de Economía y Competitividad de España, ayuda FIS2012-38244-C02-01; al Plan I2C Programa de Apoyo a la etapa Predoctoral y al Programa de Becas y Ayudas al estudio para estudiantes de enseñanzas postobligatorias, del Ministerio de Educación, Cultura y Deporte. 